\title{
Perfil do desenvolvimento motor de crianças entre 9 e 11 anos com baixo rendimento escolar da rede municipal de Maceió, AL
}

\author{
Maria Natália Santos da Silva ${ }^{a}$, Alessandra Bonorandi Dounis ${ }^{b}$ \\ ${ }^{a}$ Núcleo de Terapia Ocupacional, Universidade Federal de Sergipe - UFS, Lagarto, SE, Brasil

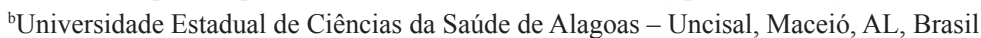

\begin{abstract}
Resumo: Introdução: Ao longo do seu desenvolvimento, a criança pode apresentar atrasos motores que, consequentemente, podem influenciar no seu processo de aprendizado. Dessa forma, destaca-se a necessidade de uma avaliação específica para detectar precocemente tais atrasos numa tentativa de dirimir ou amenizar possíveis prejuízos associados. Objetivo: Traçar o perfil do desenvolvimento motor em crianças entre 9 e 11 anos com baixo rendimento escolar da rede municipal de ensino da cidade de Maceió, AL. Metodologia: Este artigo caracteriza-se por um estudo exploratório, descritivo, transversal, que utiliza a Escala de Desenvolvimento Motor (EDM) para analisar os principais componentes de desempenho. Foram realizadas avaliações com 43 crianças de ambos os sexos. Resultados: A crianças apresentaram perfis motores que variaram de "normal médio" a "muito inferior", corroborando achados na literatura. Conclusões: Os resultados obtidos convergem com a literatura, apontando estreita relação entre desenvolvimento motor e baixo rendimento escolar, enfatizando a importância da intervenção psicomotora para a maturação de padrões motores mais complexos.
\end{abstract}

Palavras-chave: Desempenho psicomotor, Aprendizagem, Baixo rendimento escolar.

\section{Motor development profile in 9-11 year-old children from the municipal education system of Maceio, Alagoas State, presenting low school performance}

\begin{abstract}
Introduction: Children may present motor development delays that can influence their learning process, hence the need for specific assessment for the early detection of such delays in an attempt to resolve or mitigate possible future damage. Objective: Profile of motor development in children aged 9-11 years old presenting low academic achievement in the municipal education system of Maceio, Alagoas state. Methodology: An exploratory, descriptive, transversal study which uses the Motor Development Scale (MDS) to analyze the main components of performance. Evaluations were carried out with 43 children of both genders. Results: The children assessed presented motor profiles ranging from "normal" to "far below average", corroborating the findings in the literature. Conclusions: The results obtained are in agreement with the literature, showing a close relation between motor development and low school performance, emphasizing the importance of psychomotor intervention for the maturation of more complex motor patterns.
\end{abstract}

Keywords: Psychomotor performance, learning, underachievement. 


\section{Introdução}

O desenvolvimento infantil é marcado por profundas transformaçóes, refletidas em aquisições cognitivas e habilidades motoras. Partindo desta premissa, Galvão (2000) identifica a existência de etapas claramente diferenciadas no desenvolvimento humano, caracterizadas por um conjunto de necessidades e interesses que lhe garantem coerência e unidade. Ocorrem numa ordem necessária, sendo cada uma a preparação indispensável para o surgimento das seguintes. Conforme a idade, a criança interage mais fortemente com um ou outro aspecto do seu contexto, retirando dele recursos para o seu desenvolvimento.

Nessa direção, compreendemos que:

todo desenvolvimento motor efetiva-se sempre sob uma ideal adaptação aos estímulos externos. Organismo e meio ambiente são dependentes um do outro nesse conjunto de normas. A motricidade possibilita ao homem o confronto com o meio ambiente. Para a criança, a melhora constante das capacidades motoras significa a aquisição da sua independência e a capacidade de se adaptar a fatos sociais. As fases motoras e os processos psíquicos e cognitivos influenciam-se reciprocamente de modo imediato, manifestando-se quase sempre mediante modalidades comportamentais motoras como, por exemplo, pela mímica ou por meio da atitude corporal; daí terem função de comunicação com o meio ambiente (FLEHMING, 2005, p. 9).

Cada etapa do desenvolvimento é marcada por características próprias que conferem a maturidade necessária àquela idade cronológica. Nesse contexto, Burns (1999) descreve que crianças na faixa etária de 9 a 11 anos de idade possuem movimentos cada vez mais controlados, mais fluentes e mais rápidos, além de se tornarem mais automáticos e de execução mais fácil. Suas combinaçóes e sequências passam também a ser mais rápidas. Dispóem de controle eficaz sobre os padrões motores fundamentais e já apresentam pega dinâmica em tripé perfeitamente desenvolvida e bom controle postural quando sentadas. Além disso, os movimentos delicados melhoram nos mesmos parâmetros que os movimentos grosseiros. Cita ainda outras habilidades como: melhoria do controle sobre o lápis, refinamento da sensibilidade cinestésica e dissociação dos movimentos. É também nessa fase que se inicia o estágio categorial que, segundo Wallon, é caracterizado pelo interesse nas coisas e conquista do mundo exterior (GALVÃO, 2000).
Nesse sentido é possível dividir a faixa etária entre 9 e 11 anos da seguinte forma:

- 9 a 10 anos - Automatização dos movimentos habituais até se tornarem ágeis, mais relaxado na postura, levanta alternativas para solucionar problemas, relaciona-se cooperativamente com a comunidade, explica conceitos abstratos, fala e age ao mesmo tempo;

- 11 anos - Combina simultaneamente os movimentos dos membros inferiores e superiores, equilibra força muscular e habilidade, movimentos expressivos faciais e corporais, atividades com diferenciação dos sexos e desenvolvimento muscular maior nos meninos (BUENO, 1998)

Entretanto, nem sempre o desenvolvimento segue um fluxo contínuo. A criança pode apresentar alterações motoras que, consequentemente, podem influenciar seu desempenho cognitivo, refletindo em dificuldade de aprendizagem.

Contribuindo para esse debate, Ferreira (2007) afirma que as modificaçóes do sistema nervoso da criança durante seu crescimento, assim como suas formas de comportamento, caracterizam a aprendizagem.

Nessa direção, Amaro et al. (2009) descrevem que entre as causas mais frequentes das dificuldades de aprendizagem encontram-se: atraso global do desenvolvimento psicomotor; déficits sensoriais da visão e audição; doenças crônicas, que obrigam a ausências prolongadas da escola; dificuldades de aprendizagem resultantes da vivência num meio socioeconômico muito débil; dificuldades provocadas por situações de perturbação emocional e as perturbaçóes específicas do desenvolvimento (perturbação da atenção e hiperatividade, perturbaçóes da leitura e do cálculo, atraso específico da linguagem ou perturbação da coordenação motora).

Nessa perspectiva, entende-se que:

dificuldades de aprendizagem estáo diretamente ligadas às perturbações psicomotoras, que tendem a refletir-se na adaptação, orientação, direção e representação espacial, no processamento sequencial rítmico e nos problemas de lateralidade perceptivo-simbólica. Esses aspectos são apontados como variáveis determinantes nos testes que medem os problemas nas aprendizagens básicas (NETO; COSTA, 2001, p. 1).

Sendo assim, Amaro et al. (2009) certificam que a educação motora tem influência no desenvolvimento 
de crianças com dificuldades escolares, como problemas de atenção, leitura, escrita, cálculo e socialização, e destaca que a importância de um bom desenvolvimento psicomotor para a educação do corpo, bem como para o desenvolvimento global da pessoa, reside em seu papel preventivo nas dificuldades escolares.

Alguns estudos confirmaram uma estreita relaçáo entre atraso no desenvolvimento e baixo rendimento escolar. Destacam-se os de Rosa Neto (1996), em que o autor apurou que crianças com dificuldade de aprendizagem apresentaram desenvolvimento motor inferior à normalidade em relação ao grupo controle, e os de Sanches et al.(2004), que também estudou crianças com dificuldades na aprendizagem e verificou que esse fator pode influenciar na aprendizagem escolar.

Nessa direção, Costa (2001) consideram que as dificuldades de aprendizagem estão diretamente ligadas às perturbaçốes psicomotoras que tendem a refletir-se na adaptação, orientação, direção e representação espacial, no processamento sequencial rítmico e nos problemas de lateralidade perceptivosimbólica. Esses aspectos são apontados como variáveis determinantes nos testes que medem os problemas nas aprendizagens básicas.

Entretanto percebe-se que o convívio escolar permite que a criança esteja em constante troca de informaçóes nas facetas do seu desenvolvimento biopsicossocial. Por conseguinte, a experiência das crianças na escola atinge e é atingida por todos os aspectos do seu desenvolvimento, sejam eles cognitivos, motores, emocionais ou sociais, que são influenciados entre si no decorrer do crescimento do indivíduo.

Estudos sobre a motricidade infantil, em geral, são realizados com objetivo de conhecer melhor as crianças e de poder estabelecer instrumentos de confiança para avaliar, analisar e estudar o desenvolvimento de alunos em diferentes etapas evolutivas (ROSA NETO, 2002).

Santos (2006) destaca a importância da avaliação do desenvolvimento motor geral dessas crianças no diagnóstico de possíveis atrasos, evitando assim complicaçóes que possam vir a ocorrer futuramente, conhecendo precocemente as alteraçóes nos pré-escolares, traçando melhores objetivos e técnicas para a população analisada. Quanto mais precocemente forem identificadas tais alteraçóes, melhor será o desenvolvimento motor da criança.

Dessa forma, a utilização de testes para avaliar o desenvolvimento de escolares é relevante, pois permite conhecer melhor seu perfil e identificar possíveis problemas, contribuindo de forma preventiva e reeducativa, a fim de amenizar ou afastar fatores inibidores do potencial de aprendizagem da criança.

As formas de avaliar o desenvolvimento motor de uma criança podem ser diversas, no entanto nenhuma é perfeita nem engloba holisticamente todos os aspectos do desenvolvimento. Nessa perspectiva, Rosa Neto (2002) propóe uma Escala de Desenvolvimento Motor (EDM) composta por uma bateria de testes para avaliar o desenvolvimento motor de crianças dos 2 aos 11 anos de idade. O conjunto de testes ou de provas utilizado para avaliar várias características motoras de um indivíduo é chamado bateria motora (ROSA NETO, 2002). A EDM compreende um conjunto de provas muito diversificadas e de dificuldade graduada, conduzindo a uma exploraçáo minuciosa de diferentes setores do desenvolvimento, permitindo avaliar o nível de desenvolvimento motor da criança de acordo com a IC (idade cronológica), considerando êxitos e fracassos. Essa escala compreende testes motores nos seguintes componentes: motricidade fina, motricidade global, equilíbrio, esquema corporal, organização espacial e temporal.

Santos (2006) resume a motricidade fina como a capacidade de controlar um conjunto de atividades de movimento de certos segmentos do corpo, com emprego de força mínima, a fim de se atingir uma resposta precisa à tarefa.

As áreas do desenvolvimento motor avaliadas na EDM são descritas da seguinte forma:

- Desenvolvimento motor fino ou adaptativo é representado pelo uso dos pequenos músculos, da mão e da coordenaçáo olho/mão, resultando na capacidade de manipular pequenos objetos, escrever, desenhar e de toda a gama de açóes que exigem destreza manual;

- Motricidade global envolve a habilidade de dominar as contraçôes dos grandes músculos corporais na geração de movimentos amplos. Essas habilidades correspondem às capacidades como, por exemplo, sentar, andar e correr;

- O equilíbrio é a capacidade do organismo de assumir e sustentar qualquer posição contra a força da gravidade, de forma que todas as forças que agem sobre esse corpo sejam anuladas;

- O esquema corporal refere-se à capacidade de discriminar com exatidão as partes corporais, sustentar ativamente todos os gestos que o corpo realiza sobre si mesmo e sobre os objetos exteriores e organizar as partes do corpo na execução de uma tarefa; 
- Organização espacial é o conhecimento das dimensôes corporais, tanto o espaço do corpo como o espaço circundante, e a habilidade de avaliar com precisão a relação entre o corpo e o ambiente;

- Organização temporal refere-se à consciência do tempo que se estrutura sobre as mudanças percebidas e caracteriza-se pela ordem, distribuição cronológica e pela duração dos eventos (ROSA NETO, 2002).

A EDM é indicada para crianças com dificuldades de aprendizagem escolar, atrasos no desenvolvimento neuropsicomotor, problemas na fala, na escrita e no cálculo, problemas de conduta, alteraçôes neurológicas, mentais, sensoriais etc. Consiste em testes que avaliam as habilidades motricidade fina, motricidade global, equilíbrio, esquema corporal, organização espacial e organização temporal, fornecendo a idade e o quociente motor geral e as idades e os quocientes motores nessas áreas específicas, além de identificar a lateralidade (mãos, olhos e pés). Os escores dos quocientes motores são correlacionados em níveis: muito superior, superior, normal alto, normal médio, normal baixo, inferior e muito inferior.

Diante do exposto, o objetivo geral deste estudo foi traçar o perfil do desenvolvimento motor em crianças entre 9 e 11 anos com baixo rendimento escolar da rede municipal de ensino da cidade de Maceió, AL, e seu objetivo específico foi identificar se há relaçáo entre o baixo rendimento escolar e o atraso no desenvolvimento motor comparando sexo, idade e série nos diferentes componentes psicomotores.

\section{Procedimentos metodológicos}

O presente estudo foi submetido e aprovado pelo Comitê de Ética em Pesquisa com seres humanos da Universidade Estadual de Ciências da Saúde de Alagoas - Uncisal (Protocolo 1057).

Trata-se de um estudo exploratório, descritivo, com delineamento transversal, cuja amostra foi escolhida por conveniência, constituída por 43 alunos de ambos os gêneros que se enquadravam nos seguintes critérios de inclusão: ter entre 9 e 11 anos de idade, estar matriculado na terceira ou quarta série do ensino fundamental, frequentar regularmente a escola e apresentar histórico de baixo rendimento escolar há, no mínimo, um ano e/ou repetência comprovada através da ata de resultados finais.

As três escolas públicas municipais são situadas na II Região Administrativa de Maceió e foram escolhidas aleatoriamente por pertencerem à região vicinal da Uncisal. O rendimento escolar foi baseado na média bimestral das escolas municipais, que é de 6,0 pontos, calculada de acordo com a média global do aluno, que representa a média aritmética de todas as disciplinas.

Sendo assim, os alunos que se enquadraram nos respectivos critérios foram pré-selecionados e os pais ou responsáveis foram convidados a comparecer à escola para uma reuniāo, onde foi realizada a leitura e esclarecimento do Termo de Consentimento Livre e Esclarecido (TCLE). Somente após o consentimento da diretoria e dos pais ou responsáveis teve início a coleta de dados, na qual as crianças foram avaliadas em locais amplos, fechados, bem iluminados e ventilados, livres de ruídos e interrupçóes exteriores, dentro da própria escola, durante o horário de aula e respeitando-se o calendário escolar, num procedimento de coleta individual com duração de 30 a $40 \mathrm{~min}$.

Para a avaliação do desenvolvimento motor foi utilizada a Escala de Desenvolvimento Motor - EDM desenvolvida e validada por Rosa Neto (2002). A referida escala envolve um conjunto de provas diversificadas e específicas para cada faixa etária (2 a 11 anos), em cada elemento da motricidade (motricidade fina, motricidade global, equilíbrio, esquema corporal, organização espacial, organização temporal e lateralidade). A complexidade da prova a ser realizada aumenta de acordo com a idade e só termina quando a criança não consegue bom desempenho, identificando assim sua idade motora (IM) naquele componente, que será positiva se for superior à idade cronológica e negativa se for inferior. A pontuação da escala oferece uma idade motora geral (IMG), obtida através da soma dos resultados positivos obtidos nas provas motoras (IM1+IM2 ...+IM6 dividido por seis), onde os valores são expressos em meses. Para classificar o desenvolvimento motor, calcula-se o quociente motor geral (QMG) por meio da divisão entre a IMG e a idade cronológica (IC) e multiplica-se o resultado por 100. O resultado desse cálculo deve ser comparado aos números tabelados da escala (encontrada no manual), o que classificará a criança nos níveis: muito inferior (69 pontos ou menos), inferior (70-79), normal baixo (80-89), normal médio (90-109), normal alto (110-119), superior (120-129) e muito superior (130 ou mais). Os resultados também podem ser inseridos em uma base de dados da própria escala (adquirida junto com o material e o manual), onde os cálculos citados são realizados automaticamente, gerando gráficos e tabelas individuais que podem ser armazenados. 
Assim, os testes motores foram aplicados na mesma ordem encontrada no Manual de Avaliação Motora (ROSA NETO, 2002), tendo início a partir da idade cronológica da criança. Entretanto, apesar de algumas crianças terem alcançado todas as etapas de um ou mais itens da avaliação, outras apresentaram maiores dificuldades, sendo necessário iniciar o teste a partir de uma idade muito inferior à sua idade cronológica.

Nessa escala, teste motor é entendido como uma prova específica que permite medir uma determinada característica motora de um indivíduo e comparar seus resultados com os valores considerados normais de acordo com a escala. Os resultados de uma prova motora permitem determinar o avanço ou atraso motor de uma criança naquele aspecto avaliado. A diferença entre a idade cronológica e a idade motora definirá se a criança tem ou náo atraso motor. Dessa forma, se os valores forem negativos (idade negativa) significa que a idade motora geral é menor que a idade cronológica da criança, caracterizando, segundo a escala, atraso motor.

A aplicação em um sujeito permite avaliar seu nível de desenvolvimento motor, considerando êxitos e fracassos, levando em conta as normas estabelecidas pelo autor da escala. Em cada prova motora foi selecionado o aspecto diferencial de outras provas, respaldado por autores clássicos como Zazzo, Piaget, Vayer, Guilman, Ozerestsky, Granjon e Stambak e por diversos testes motores e psicológicos existentes.

Após observação minuciosa em cada prova, os resultados foram analisados por meio da idade motora (IM) obtida em cada item, no qual as análises foram realizadas com todos os pontos válidos por prova motora, uma vez que algumas crianças não conseguiram realizar todas as provas.

\section{Resultados e discussão}

Participaram do estudo 19 crianças do sexo feminino $(44,1 \%)$ e 24 do sexo masculino $(55,8 \%)$. Dessas, 19 frequentavam a quarta série $(44,1 \%)$ e 24, a terceira série $(55,8 \%)$. A distribuição das idades ficou definida em: duas crianças com 9 anos, 18 com 10 e 23 com 11 anos, perfazendo a maioria.

De maneira geral, os escolares apresentaram padrốes motores distribuídos em níveis "normal médio" (11,6\%), "normal baixo" (44,1\%), "inferior" $(37,2 \%)$ e "muito inferior" (6,9\%), de acordo com a EDM. Dessa forma, o quociente motor geral da amostra foi classificado em níveis "normal baixo" e "inferior", conforme ilustra o gráfico 1 . Três componentes classificaram-se em nível "normal médio", a saber: Motricidade global, Esquema corporal e Organização temporal. Motricidade fina e Organização espacial classificaram-se em "inferior" e "muito inferior", respectivamente. O componente equilíbrio foi classificado em "normal baixo".

As dificuldades no aprendizado muitas vezes são refletidas em alteraçóes de ordem psicomotora. Fiates (2001) apurou que pesquisadores como Wallon, Piaget, Vayer, Le Boulch e Fonseca comprovam que existe estreita ligação entre o movimento e o processo de desenvolvimento e aprendizagem da criança, destacando o papel da psicomotricidade como determinante na dialética do desenvolvimento humano.

A classificação dos resultados segundo a EDM demonstrou que $88,2 \%$ das crianças com baixo rendimento escolar avaliadas apresentaram níveis motores abaixo do padráo esperado para a idade cronológica. Tendo em vista esses resultados, podemos inferir que esses dados podem indicar uma relação ente baixo rendimento escolar e atrasos do desenvolvimento motor, corroborando achados de outros estudos (ROSA NETO, 1996; COSTA, 2001; ROSA NETO et al., 2004; FIATES, 2001).

De acordo com os parâmetros motores das crianças deste estudo, observa-se que grande parte da amostra apresentou idade motora inferior à idade cronológica (idade negativa), numa média de 25,3 meses. Esse achado é superior aos encontrados por Batistella (2001) - 6,6 meses, Rosa Neto (1996) - 13,4 meses, Fiates (2001) - 16,2 meses e Amaro et al. (2009) - 24,77 meses, indicando atraso motor relevante nos escolares deste estudo e enfatizando a relaçáo entre atraso no desenvolvimento motor e baixo rendimento escolar, interrogando a necessidade de um acompanhamento mais específico e eficaz nesses casos, seja na escola ou mesmo individualmente, através de encaminhamento para psicopedagogos e/ou terapeutas ocupacionais.

De maneira geral, em relaçáo ao sexo houve semelhança nos resultados que indicaram grandes dificuldades nos componentes de Motricidade fina e Organização espacial, resultado semelhante ao estudo desenvolvido por Batistella (2001). A média geral de atraso motor entre os sexos variou em quatro meses, considerada pouco relevante para a EDM.

Assim, em termos percentuais ambos os sexos apresentaram as mesmas classificaçóes em quase todas as variáveis, exceto na variável equilíbrio, de forma que o sexo feminino classificou-se em nível "normal baixo" e o masculino em "inferior". Verificou-se média de 25,3 meses de atraso motor em relação à idade cronológica (idade negativa). $\mathrm{O}$ 
sexo feminino apresentou média de 22,7 meses e o masculino obteve média de 26,6 meses de atraso motor, conforme ilustra a Tabela 1.

Esse resultado contradiz os estudos realizados por Thomas e French (1985) apud Burns (1999, p. 185), que relatam que as tarefas de equilíbrio são tão bem executadas por meninos quanto por meninas nessa idade. Em contrapartida, assemelha-se com os achados de autores como Fiates (2001) e Rosa Neto et al.( 2007).

Segundo revisão de literatura, alguns estudos mostram que:

[...] as diferenças no desempenho dos meninos e meninas de tenra idade são geralmente pequenas, podendo ser explicadas por influências culturais e sociais, visto que se tornam insignificantes diante de maior exposição e treinamento, assim como da modificação das atitudes dos adultos que lidam com as crianças de baixa idade (WALTER, 1999, p. 54).

Ainda em relação ao sexo, Eckert (1993) relata que geralmente não são feitas comparaçóes entre o desempenho motor de meninos e meninas porque são sensíveis as diferenças, existindo somente alguns eventos em que um dos sexos tende a aumentar em relação ao outro, como, por exemplo, nas atividades que requerem força, nas quais os meninos são superiores.

Em relação às séries houve pouca diferença nos percentuais das classificaçóes gerais. A terceira série apresentou resultados iguais em dois perfis motores, a saber: "normal baixo" (23,25\%) e "inferior" (23,25\%). Já a quarta série foi classificada como "normal baixo" (23,25\%). Assim, as classificaçóes dos resultados obtidos na escala, de forma geral, foram semelhantes com relação às séries, já que ambas apresentaram perfis motores classificados como "normal baixo". Entretanto, a terceira série também apresentou perfil classificado em "inferior". Esses resultados são opostos aos encontrados por Batistella (2001), para ele os alunos da quarta série apresentaram valores inferiores às outras séries em relação aos quocientes motores.

Com relação à idade, as crianças de 9 anos classificaram-se cada uma em um perfil, sendo eles "normal médio" e "normal baixo". A maioria das crianças de 10 anos apresentou resultados classificados no perfil "normal baixo" (44,4\%). Já as crianças de 11 anos apresentaram níveis "normal baixo" (47,8\%) e "inferior" (43,4\%), apresentando pouca diferença entre os perfis. As idades negativas encontradas de acordo com a idade, da menor para a maior, foram: 14,5 meses, 23,9 meses e 27,4 meses. Esses dados podem ser melhor visualizados na Tabela 2 .

As principais dificuldades encontradas nas crianças de 10 e 11 anos foram nos componentes: "organização espacial", no qual o perfil foi classificado como "muito inferior", com $77,7 \%$ e $69,5 \%$, respectivamente, e "motricidade fina", cujo perfil foi classificado como "inferior" com 55,5\% e 43,4\%, respectivamente. Particularmente o perfil das crianças de 11 anos variou entre "inferior" e "normal médio" na variável "motricidade global”, com 34,7\% e 30,4\%, respectivamente. Segundo Bee (2003), as habilidades motoras amplas se desenvolvem mais cedo e as finas mais tarde. Entretanto, ao contrário dessa afirmativa, algumas crianças de 11 anos apresentaram déficit na motricidade global, confirmando novamente um atraso motor.

No que concerne à lateralidade, os resultados observados mostram que $53,4 \%$ das crianças

Tabela 1. Classificação segundo a EDM em relação ao sexo

\begin{tabular}{|c|c|c|c|c|}
\hline Prova/Componente & N. Médio & N. Baixo & Inferior & M. Inferior \\
\hline 1 - Motricidade fina & $\begin{array}{l}7=36,84 \% * \\
7=\mathbf{2 9}, \mathbf{1 6} \%\end{array}$ & $\begin{array}{c}3=15,78 \% \\
\mathbf{6}=\mathbf{2 5 \%}\end{array}$ & $\begin{array}{c}8=42,10 \% \\
\mathbf{1 1}=\mathbf{4 5 , 8 3}\end{array}$ & $\begin{array}{c}1=5,26 \% \\
-\end{array}$ \\
\hline 2 - Motricidade global & $\begin{array}{l}7=36,84 \% \\
\mathbf{8}=\mathbf{3 3 , 3 3 \%}\end{array}$ & $\begin{array}{l}5=26,31 \% \\
\mathbf{8}=\mathbf{3 3 , 3 3 \%}\end{array}$ & $\begin{array}{l}5=26,31 \% \\
\mathbf{7}=\mathbf{2 9 , 1 6 \%}\end{array}$ & $\begin{array}{l}2=10,52 \% \\
1=4,16 \%\end{array}$ \\
\hline 3 - Equilíbrio & $\begin{array}{l}7=36,84 \% \\
\mathbf{3}=\mathbf{1 2 , 5 \%}\end{array}$ & $\begin{array}{c}5=26,31 \% \\
\mathbf{6}=\mathbf{2 5 \%}\end{array}$ & $\begin{array}{l}5=26,31 \% \\
\mathbf{8}=\mathbf{3 3 , 3 3 \%}\end{array}$ & $\begin{array}{l}2=10,52 \% \\
\mathbf{7}=\mathbf{2 9 , 1 6 \%}\end{array}$ \\
\hline 4 - Esquema corporal & $\begin{array}{l}10=52,63 \% \\
\mathbf{8}=\mathbf{3 3}, \mathbf{3 3} \%\end{array}$ & $\begin{array}{c}3=15,78 \% \\
\mathbf{6}=\mathbf{2 5} \%\end{array}$ & $\begin{array}{c}5=26,31 \% \\
\mathbf{6}=\mathbf{2 5 \%}\end{array}$ & $\begin{array}{l}3=15,78 \% \\
\mathbf{4}=\mathbf{1 6 , 6 6 \%}\end{array}$ \\
\hline 5 - Organização espacial & -- & $1=4,16 \%$ & $\begin{array}{c}6=31,57 \% \\
\mathbf{6}=\mathbf{2 5} \%\end{array}$ & $\begin{array}{l}13=68,42 \% \\
\mathbf{1 7}=\mathbf{7 0 , 8 3} \%\end{array}$ \\
\hline 6 - Organização temporal & $\begin{array}{l}8=42,10 \% \\
\mathbf{8}=\mathbf{3 3}, \mathbf{3 3} \%\end{array}$ & $\begin{array}{c}5=26,31 \% \\
\mathbf{6}=\mathbf{2 5 \%}\end{array}$ & $\begin{array}{l}2=10,52 \% \\
\mathbf{7}=\mathbf{2 9 , 1 6 \%}\end{array}$ & $\begin{array}{c}4=21,05 \% \\
\mathbf{3}=\mathbf{1 2}, \mathbf{5} \%\end{array}$ \\
\hline
\end{tabular}

*As porcentagens correspondem ao resultado dividido pela quantidade de meninos/meninas separadamente; legenda: negrito $=$ masculino 
Tabela 2. Classificação segundo a EDM em relação à idade

\begin{tabular}{ccccc}
\hline Idade & N. Médio & N. Baixo & N. Inferior & M. Inferior \\
\hline 9 anos & $1=50 \%$ & $1=50 \%$ & - & - \\
10 anos & $3=16,6 \%$ & $8=44,4 \%$ & $6=33,3 \%$ & $1=5,5 \%$ \\
11 anos & $1=4,34 \%$ & $11=47,82 \%$ & $10=43,47 \%$ & 1 \\
\hline
\end{tabular}

eram destras completas, $4,6 \%$ sinistras completas e $44,1 \%$ tinham a lateralidade cruzada. Não houve lateralidade indefinida. Segundo Bueno (1998), a criança entre 7 e 8 anos já é capaz de reconhecer a lateralidade no outro e as habilidades psicomotoras são amplamente exploradas nos esportes e atividades físicas. Contradizendo essa afirmativa, os resultados demonstram que as principais dificuldades encontradas pela maioria das crianças estudadas, independentemente da idade, referem-se à lateralidade, na qual foi possível notar grande dificuldade na realização das provas nesses aspecto, ou mesmo náo conseguiram concluir a atividade com êxito.

Para Caetano, Silveira e Gobbi (2005), a exploração do ambiente e das próprias potencialidades da criança geram experiências que podem afetar o índice de aparecimento de certos padróes de comportamento, privilegiando mais um componente da motricidade do que outro. Além disso, o contexto ou ambiente em que as crianças estáo inseridas e as exigências das tarefas propostas influenciam grandemente o aparecimento de novas habilidades. Dentro dessa premissa, observa-se que os maiores déficits encontrados foram nas áreas Motricidade fina e Organização espacial, com perfis motores classificados como "inferior" e "muito inferior", respectivamente, semelhante aos resultados encontrados por Amaro et al. (2009) que, além desses componentes, observaram atraso significativo na organização temporal.

Nessa direção, uma observação importante de se destacar é que nas três escolas há carência de brinquedos, parques e área livre para recreação, o que pode ter refletido nos resultados negativos obtidos. Cabe, portanto, destacar a importância de um ambiente rico e estimulador nas escolas, a fim de contribuir para um melhor desenvolvimento dessas crianças.

A distribuição da classificação da preferência lateral entre os escolares estudados apontou maior prevalência para destro completo, seguido de cruzada e sinistro completo, corroborando achados de outras pesquisas como de Rosa Neto (1996) e Santos (2006), que mostram uma predominância da lateralidade destra completa e cruzada. Não houve lateralidade indefinida, justificada pelo fato de que a preferência por uma das mãos, embora já possa ser verificada aos 3 anos de idade, geralmente torna-se evidente dos 4 aos 5 anos (PEREIRA, 2001).

Os dados encontrados neste estudo revelam que os educandos com baixo rendimento escolar apresentam também importante atraso no desenvolvimento motor, sugerindo uma estreita relação entre esses dois componentes, conforme aponta a literatura.

Dessa forma, esses achados contribuem para uma atuação mais específica de profissionais da área e afins destacando a relevância da intervenção precoce em atrasos motores, principalmente no início da vida escolar, a fim de identificar possíveis intervençôes mais específicas em cada caso, contribuindo para um melhor desempenho motor e auxiliando no processo de aprendizagem da criança.

\section{Conclusão}

Sabe-se que o desenvolvimento motor nem sempre segue uma sequência linear, podendo haver um comprometimento em algum de seus componentes, variando entre as pessoas no que se refere ao grau de prejuízo. Vários autores consideram que as alteraçôes no desenvolvimento motor são percebidas como um componente que interfere no desenvolvimento biopsicossocial, evidenciando, desse modo, problemas afetivos, de aprendizagem, de relação consigo mesmo e com os outros e problemas vinculados à vivência do corpo.

Os resultados do estudo verificaram que as crianças avaliadas apresentaram perfil motor classificado em "normal baixo", inferindo que crianças com dificuldade de aprendizagem também apresentam atraso em seu desenvolvimento motor, como aponta a literatura. Dessa forma, é importante destacar a necessidade da utilização de testes para avaliar o desenvolvimento de escolares, permitindo conhecer melhor seu perfil e intervir precocemente através da elaboração de programas que aperfeiçoem as habilidades indispensáveis para a aquisição de outros padrôes motores mais complexos, estimulando sua maturação psicomotora e, consequentemente, facilitando seu processo de aprendizagem. 


\section{Referências}

AMARO, K. N. et al. Desenvolvimento motor em escolares com dificuldade de aprendizagem. Revista Digital, Buenos Aires, v. 14, n. 133, p. 45-51, 2009.

BATISTELLA, P. A. Estudo de parâmetros motores em escolares com idade de 6 a 10 anos da cidade de Cruz Alta-RS. 2001. 103 f. Dissertação (Mestrado em Ciências do Movimento Humano)-Centro de Ciências da Saúde e do Esporte, Universidade do Estado de Santa Catarina, Florianópolis, 2001.

BEE, H. A criança em desenvolvimento. 9. ed. São Paulo: Artmed, 2003. PMCid:PMC1422713.

BUENO, J. M. Psicomotricidade: teoria e prática. São Paulo: Editora Lovise, 1998.

BURNS, Y. Desenvolvimento motor dos 2 aos 6 anos de idade. In: BURNS, Y. R.; MacDONALD, J. Fisioterapia e crescimento na infância. Sáo Paulo: Santos editora e livraria, 1999. p. 43-45.

CAETANO, M. J. D.; SILVEIRA, C. R. A.; GOBBI, L. T. B. Desenvolvimento motor de pré-escolares no intervalo de 13 meses. Revista Brasileira de Cineantropometria \& Desempenho Humano, Florianópolis, v. 7, p. 5-13, 2006.

COSTA, S. H. Perfil motor de escolares de 05 a 14 anos com dificuldades na aprendizagem. 2001. 60 f. Monografia (Graduação em Educação Física)-Centro de Ciências da Saúde e do Esporte, Universidade do Estado de Santa Catarina, Florianópolis, 2001.

ECKERT, H. M. Desenvolvimento motor. 3. ed. São Paulo: Manole, 1993.

FERREIRA, J. R. P. Saúde escolar: aspectos biopsicossociais de crianças com dificuldade de aprendizagem. 2007. 114 f. Dissertação (Mestrado em Ciências do Movimento Humano)-Universidade do Estado de Santa Catarina, Florianópolis, 2007.

FIATES, M. P. Estudo da relação entre o desenvolvimento psicomotor e as dificuldades na aprendizagem de um grupo de crianças de 4 a 7 anos. 2001. 64 f. Monografia (Especialização em Desenvolvimento Infantil)Universidade do Estado de Santa Catarina, Florianópolis, 2001.
FLEHMING, I. Texto e atlas do desenvolvimento normal e seus desvios no lactente: diagnóstico e tratamento precoce do nascimento até o $18^{\circ}$ mês. São Paulo: Atheneu, 2005.

GALVÃO, I. Henri Wallon: uma concepção dialética do desenvolvimento infantil. 8. ed. Rio de Janeiro: Editora Vozes, 2000.

PEREIRA,C. O. Estudo de parâmetros motores em pré-escolares do ensino fundamental. 2001. $74 \mathrm{f}$. Dissertação (Mestrado em Ciências do Movimento Humano)-Universidade do Estado de Santa Catarina, Florianópolis, 2001.

ROSA NETO, F. R. Manual de avaliação motora. Porto Alegre: Artes Médicas, 2002. PMid:12105118.

ROSA NETO, F. R. Valoración del desarrollo motor y su correlación con los trastornos del aprendizaje. 1996. $346 \mathrm{f}$. Tese (Doutorado em Medicina do Esporte)-Universidade de Zaragoza, Zaragoza, 1996.

ROSA NETO, F. R. et al. Desenvolvimento Motor de Crianças com Indicadores de Dificuldades na Aprendizagem Escolar. Revista Brasileira de Ciência e Movimento, Brasília, v. 15, n. 1, p. 45-51, 2007.

ROSA NETO, F. R. et al. Perfil motor em crianças avaliadas em um Programa de Psicomotricidade. Temas sobre Desenvolvimento, v. 13, n. 74, p. 19-24, 2004.

SANCHES, S. O. et al. Perfil psicomotor associado à aprendizagem escolar. Revista Digital, Buenos Aires, v. 10, n. 79, p. 1-5, 2004.

SANTOS, C. F. Z. Estudo comparativo do desenvolvimento neuropsicomotor e perfil psicossocial de crianças pré-escolares entre 03 e 05 anos de idade dos Centros educacionais infantis Mickeylândia e Pirulito de Termas do Gravatal-SC. 2006. 70 f. Monografia (Graduação em Fisioterapia)-Universidade do Estado de Santa Catarina, Florianópolis, 2006.

THOMAS, J. R.; FRENCH, K. E. Gender differences across age in motor performance: a meta-analysis. Psychological Bulletin, Washington, v. 98, n. 2, p. 260-82, 1985. http://dx.doi.org/10.1037/0033-2909.98.2.260

WALTER, P. Desenvolvimento motor do início da idade escolar à puberdade. In: BURNS,Y. R.; MacDONALD, J. Fisioterapia e crescimento na infância. São Paulo: Santos editora e livraria, 1999. p. 49-56.

\section{Contribuição dos Autores}

Ambas foram responsáveis por todas as fases da pesquisa e elaboração do texto, sendo a segunda autora a orientadora do trabalho original. 